\title{
Effects of a complex intervention on fall risk in the general practitioner setting: a cluster randomized controlled trial
}

This article was published in the following Dove Press journal:

Clinical Interventions in Aging

16 August 2013

Number of times this article has been viewed

\author{
Ellen Freiberger' \\ Wolfgang A Blank ${ }^{2}$ \\ Johannes Salb' \\ Barbara Geilhof ${ }^{3}$ \\ Christian Hentschke' \\ Peter Landendoerfer ${ }^{2}$ \\ Martin Halle ${ }^{3}$ \\ Monika Siegrist ${ }^{3}$ \\ 'Institute of Sport Science and Sport \\ Universität Erlangen-Nürnberg, \\ Nuremberg, Germany; ${ }^{2}$ Institute \\ of General Practice, Technische \\ Universität München, Munich, \\ Germany; ${ }^{3}$ Department of Prevention, \\ Rehabilitation and Sports Medicine, \\ Technische Universität München, \\ Munich, Germany
}

Correspondence: PD Ellen Freiberger Institute for Biomedicine of Aging Kobergerstr. 60, 90408 Nürnberg, Germany Tel +49 9II- 5302-96162

Fax +49 9||-5302-96|5| Email ellen.freiberger@fau.de
Purpose: To study the feasibility of first, reaching functionally declined, but still independent older persons at risk of falls through their general practitioner (GP) and second, to reduce their physiological and psychological fall risk factors with a complex exercise intervention. We investigated the effects of a 16-week exercise intervention on physiological (function, strength, and balance) and psychological (fear of falling) outcomes in community-dwelling older persons in comparison with usual care. In addition, we obtained data on adherence of the participants to the exercise program.

Methods: Tests on physical and psychological fall risk were conducted at study inclusion, and after the 16-week intervention period in the GP office setting. The 16-week intervention included progressive and challenging balance, gait, and strength exercise as well as changes to behavioral aspects. To account for the hierarchical structure in the chosen study design, with patients nested in GPs and measurements nested in patients, a three-level linear mixed effects model was determined for analysis.

Results: In total, 33 GPs recruited 378 participants (75.4\% females). The mean age of the participants was 78.1 years (standard deviation 5.9 years). Patients in the intervention group showed an improvement in the Timed-Up-and-Go-test (TUG) that was 1.5 seconds greater than that showed by the control group, equivalent to a small to moderate effect. For balance, a relative improvement of 0.8 seconds was accomplished, and anxiety about falls was reduced by 3.7 points in the Falls Efficacy Scale-International (FES-I), in the intervention group relative to control group. In total, $76.6 \%(\mathrm{~N}=170)$ of the intervention group participated in more than $75 \%$ the supervised group sessions.

Conclusion: The strategy to address older persons at high risk of falling in the GP setting with a complex exercise intervention was successful. In functionally declined, communitydwelling, older persons a complex intervention for reducing fall risks was effective compared with usual care.

Keywords: complex exercise intervention, functional declined community-dwelling older persons

\section{Introduction}

In community-dwelling older persons, falls pose a major threat to function and independence. Falls are a common cause for nursing home admission and health care utilization in this population. ${ }^{1-3}$ It is a common understanding that about $30 \%$ of persons 65 years and older experience a fall at least once a year, with a high percentage of these persons even falling several times per year. ${ }^{2,4}$ The incidence of falls, as well as fall-related injuries, increases with age. ${ }^{4,5}$ Therefore, fall prevention is an important 
component in a society facing an increasing fall-related burden on the public health care system. ${ }^{6}$

Clinical trials have shown that effective fall prevention interventions include balance training in combination with strength training. ${ }^{1,6,7}$ In contrast to this evidence, the implementation of broad ranging fall prevention programs is rare, and the challenge remains to deliver the most effective intervention to the right target group of older persons. ${ }^{8}$

The implementation process for effective intervention strategies is hampered by different barriers. One important barrier is the attitude of the older person him- or herself, and the other barrier might be the pathway of the implementation process. Research has demonstrated that over $50 \%$ of older persons offered an exercise program for fall prevention refused to take part, ${ }^{9}$ and uptake rates are sometimes less than $10 \%{ }^{10,11}$ To increase the uptake rate, different strategies are feasible. One strategy could be to utilize the pathway of the general practitioner (GP) office setting to target at-risk older persons. GPs might be the key persons to motivate at-risk older persons to participate in exercise programs for multiple reasons: research has demonstrated that older persons see their GP on a regular basis, view their GP as a very important source of health-related information, and value their advice. ${ }^{12}$ GPs are mostly familiar with the daily routine and needs of their older patients, but in contrast, only few studies have investigated fall prevention programs in the GP setting. ${ }^{13}$ For German GPs, the Geriatric recommendations dictate that $^{14}$ in persons 65 years and older, fall history during the past 6 months should be assessed at least once each year. A recent study in Germany found that $83 \%$ of the GPs were unaware of the recent falls of their patients in the previous 6 months. ${ }^{15}$ These results illustrate the need to include GPs in fall prevention research targeting older persons at high risk of falling.

In this paper, we investigated the effects of a previously validated 16-week complex exercise intervention targeting community-dwelling older persons at high risk of falling, in the GP setting. We compared the effects of the exercise program on physical and psychological fall-risk outcomes in the intervention group (IG) with the group receiving usual care. The outcomes were balance, strength, function, and fear of falling. All fall-risk outcomes were measured at the start of the study and after the 16-week intervention. In addition, we obtained data on adherence to the exercise sessions by the included older persons. Furthermore, this paper presents in-depth information on the complexity and necessity of the exercise program, including the different components of the exercise program and the challenges in the recruitment process, for both the GPs and at-risk patients.

\section{Methods}

\section{Study design}

The study protocol (PreFalls NCT01032252) we used has been previously published and no changes were made. ${ }^{16}$ In short, it was a controlled, multicenter, prospective study design with an equal cluster, random allocation of participating GPs to a complex intervention or usual care control group (CG). The effects of the complex 16-week exercise intervention on physical and psychological fall risks outcomes were investigated. The number of falls and rate of fallers at 12 and 24 months postintervention will be obtained. Participating patients of the included GPs were tested at four points: at baseline (T0); after the intervention (T1); 12 months after baseline; and 24 months after baseline. The study protocol was approved by the Ethics Board of the Faculty of Medicine of the Technische Universität München. The study was conducted according to the Helsinki Declaration. In this paper, the effects of the 16-week intervention on fall risk and the physical and psychological outcomes between T0 and T1 were reported.

\section{Participants - recruitment of GPs and cluster randomization}

GPs were recruited through local "quality" peer groups, from networks affiliated to the Institute for Family Medicine of the Technische Universität München and by the Institute of Sport Science and Sports of the University of ErlangenNuremberg, as well as through additional advertisement in German medical journals. ${ }^{16}$ GPs were invited to participate in the study by an invitation letter, and in case of no reply, contacted via telephone. All the participating GPs took part in an educational workshop lasting 3.5 hours. The workshop provided the GPs and one of their staff members with information regarding the study, including the objectives, definition of falls used in the study, and topics of the intervention. In addition, GPs and their staff members were trained in the testing procedure and management of the fallrisk assessment. GPs gained Continuing Medical Education (CME) points for attending the workshop. ${ }^{16}$ With regard to implementation of fall risk assessment in the GP setting and sustainability strategies, no incentives were provided for the GPs for study participation and testing procedures (due to the current health systems structure in Germany). After attending the educational workshop, the GPs were randomized either to 
the intervention or the $\mathrm{CG}$ and started to recruit their patients. Randomization was conducted by a blockwise randomization list for both coordination centers, by a statistician who was otherwise uninvolved in the study at the time. Neither the GP nor staff members were blinded to the randomization allocation - blinding for the testing procedure is not feasible in an exercise intervention study because participants in the exercise intervention arm would tell their GPs what they were doing. Cluster randomization was the best solution to avoid contamination of the CG. ${ }^{19}$

After the randomization process, each GP received a list with the personal identification number IDs for the recruitment of their future participants.

\section{Participants - recruitment of patients}

GPs or trained staff members in the participating practices approached eligible functionally declined, communitydwelling patients in their respective settings for participation in the study. The approach was based on self-selection as well as patients records, and final inclusion was based on the objective fall risk assessment.

The inclusion criteria were defined as: aged 65 years and older; reporting one or more falls in the past 12 months and/or fear of falling and/or physical fall risk obtained with the fall risk assessment (described further down); a cutoff score of $<10$ seconds for the strength and function tests; and self-reported or measured balance impairments. ${ }^{16}$ At least one inclusion criterion had to be fulfilled to take part in the study. All patients were required to sign informed consent forms before they were tested in their GP's office setting. ${ }^{16}$ All participants had to be mobile eg, able to stand alone and walk alone or with an assistant device. Participants could not be wheelchair-bound. Sample size calculations were based on fall reductions and showed that about 382 participants were needed. ${ }^{16}$

\section{Recruitment of the fall intervention exercise instructors}

The intervention instructor was either recruited by the participating GP or by the local study coordinator. The instructor was either an experienced physiotherapist or sports scientist. ${ }^{16}$ For standardization of the multicenter intervention, all the instructors took part in an 8-hour educational workshop, in which they were trained to apply the standardized complex exercise program, as well as given background information of the study, eg, objective of the study, tests used, and fall definition. ${ }^{16}$ In contrast to the GPs, the exercise instructors were reimbursed for their sessions, in accordance with the German national standards. To avoid testing bias, the exercise instructors were not involved in the testing procedure. Realization and the subjective experience of the instructors were obtained with a questionnaire after the exercise program ended.

\section{Testing procedure}

After signing the informed consent sheet, the eligible patient was tested by the GP or the trained staff member to ensure the inclusion criteria and obtain the base line status. In case of problems with the testing procedure due to a time restriction, the regional coordination team supported the testing process for that specific GP. All GPs collected the testing protocol and data and then sent all the documents via postal service to the regional coordination center. The regional coordination center paid for the postal service and entered the data into the common database. All data management was handled at the coordination centers, in a common database.

Testing in the IG and CG, for physical and psychological fall risk, was conducted at $\mathrm{T} 0$ and at $\mathrm{T} 1$.

The testing was split between the GPs, who managed medication and chronic diseases, and the trained staff member, who managed the questionnaires and physical performance tests. Each GP setting had one trained staff member who was responsible for the testing procedure throughout the study and to whom the testing material was sent. Each GP received, in addition to the training, a standardized test protocol to ensure the reliability of outcome measurements.

\section{Fall risk assessment}

A series of physical performance tests was administered in the GP setting to evaluate the fall risk. ${ }^{16}$ The Timed Up and Go test (TUG) ${ }^{17}$, the five repetition Chair Stand Test $(\mathrm{CST})^{18}$, and a modified Romberg test (mod Rom) were used for physical fall risk assessment. For psychological outcomes, the German version of the Falls Efficacy Scale International (FES-I) was used. ${ }^{16}$ The TUG was performed over a 3-meter course, and staff members had to note the use of walking aids in the testing protocol. The participant was asked to walk as fast, but safely as possible. Time was taken in seconds with a stop watch. The five repetitions CST was performed with arms crossed over the chest. The time it took to stand up and sit down five times was measured in seconds by a stopwatch. ${ }^{16}$ Again, any deviation from the testing protocol had to be noted by the staff member. In case five repetitions were not possible due to the limitation of the 
participants, the numbers of chair rises had to be reported. The mod Rom measures static balance in three conditions: feet side by side, semitandem, and tandem stance, for ten seconds in each position. Fear of falling was assessed with the German FES-I. ${ }^{16}$

\section{Intervention}

The complex exercise program was based on a formerly established effective exercise program. ${ }^{20,21}$ It was developed following a biopsychosocial approach, enhancing resources in older, community-dwelling persons. ${ }^{21}$ Participating patients of the randomized intervention GPs were organized in groups of 5-15 older persons. The intervention included a combination of 28 supervised and unsupervised sessions. Sixteen sessions, once per week for 60 minutes, were supervised, and the participants added at least one unsupervised session starting from week 5. Adherence to each supervised and unsupervised session was taken with a standardized protocol and procedure by the exercise instructors. The supervised interventions took place in a community house, church location, or at the place of the exercise instructor that was near the GP setting. Each supervised session started with a short, 5-minute discussion to introduce the topic of the session and address participants' well-being and questions, followed by a 10-minute warm up phase, leading to a 30-40 minute conditioning period, followed by a 5-10 minute cooling down and closing phase with relaxation and discussion between the participants and instructors about the experience. During the exercise program, local transportation service was provided for the participants, when necessary to be able to attend the group-based sessions. The group-based intervention followed standardized protocols for comparable conditions, but with as much variety as possible to address the individual needs of participants. A third party insurance was provided to the instructors, as well as to the participants, in case of adverse events.

We defined our exercise intervention as a complex intervention due to the fact that it included several interacting components (some with behavioral aspects) on the part of the exercise instructor as well as the participants, number of groups being targeted by the exercise program, different outcomes, and a high degree of flexibility for tailoring the exercise to the participant's individual level. ${ }^{19}$ The complex exercise program (Table 1) targeted the most important fallrisk factors, balance and gait limitation and muscle strength of the upper and lower extremities. In addition, body awareness, motor coordination, self-efficacy components, and
Table I Main intervention components and their frequency per group-based intervention

\begin{tabular}{|c|c|c|c|c|c|}
\hline \multirow[t]{2}{*}{ Intervention components } & \multicolumn{4}{|c|}{ Month } & \multirow[t]{2}{*}{ Total } \\
\hline & I & 2 & 3 & 4 & \\
\hline Strength training ${ }^{\mathrm{a}}$ & 2 & 2 & I & 1 & 6 \\
\hline Challenging balance training & 2 & 2 & 2 & 2 & 8 \\
\hline Functional training ${ }^{\mathrm{b}}$ & & 1 & & & I \\
\hline Gait training & I & & I & & 2 \\
\hline Body awareness ${ }^{c}$ & & 1 & & 1 & 2 \\
\hline Fall risk education ${ }^{c}$ & & 1 & 2 & & 3 \\
\hline Home program ${ }^{d}$ & I & & & 1 & 2 \\
\hline
\end{tabular}

Notes: aprogressive upper and lower body strength exercises and stretching exercises; bfunctional training included eg, getting up from the floor; caddressing misconceptions about fall risk, attitudes about falls, thoughts and concerns about falling, negative and positive thinking patterns, and potential environmental fall hazards; dbrochure describing how to perform the strength, balance, and gait exercises safely and regularly.

small group dynamics games were included. The exercise was performed mostly in standing position. Modifications to the original exercise program ${ }^{20,21}$ were made by exercising only with body weight and without additional materials. The strength exercises were performed with increasing progression by changing frequency, speed, and range of movement. Intensity was controlled with the Borg Scale, ${ }^{22}$ an evaluated self-perceived exhaustion scale, to avoid negative events. The balance exercise included challenging conditions (eg, eyes open versus closed, reduction of base of support), and training regarding postural strategies (ankle, hip, and step strategies). The gait exercise contained different rhythmical, spatial, and temporal components, as well as a dual task condition, eg, walking and talking, and combination of gait and arm movements. At the start of the exercise program, participants were allowed to use walking aids if needed, but throughout the intervention, the use of walking aids during exercise was reduced.

To address the psychological fall risk dimension, sessions for behavioral changes and attitudes were also included in the complex exercise intervention. The sessions on psychological aspects were also part of the formerly evaluated exercise program. $^{21}$

To sum up, the complex exercise program addressed the biopsychosocial health resources of the participants and empowered their independence by including elements of patient education with regard to behavioral changes.

In order to perform the home-based unsupervised exercises, participants were provided a booklet with written instructions, safety issues, and pictures about how to do the exercise in their home. The booklet included a training schedule, which the participants could use. The exercises in the booklet were introduced in the group-based sessions and 
were occasionally integrated, as well as recalled, at the end phase of the intervention, to ensure familiarization.

To ensure comparability between the different IGs, close contacts were maintained between the local study coordinator and the instructors. After the intervention, instructors filled out a questionnaire to provide subjective information on their experience in managing the complex intervention program.

The CG did not receive any intervention but continued seeing their GPs with their usual care procedures.

\section{Statistical analyses}

The focal interest here was to investigate the effects of the applied intervention on change in general fall risk, expressed in several assessments previously described. To account for the hierarchical structure in the chosen study design with patients nested in GPs and measurements nested in patients, a three-level linear mixed-effects model was determined for analysis. Per considered outcome measure (TUG, CST, mod Rom, FES-I) one model was created, with time and IG as experimental factors. Differences in change over time were represented by the group-by-time-interaction effect, which was the primary interest. None of the four outcome models showed the third level of GPs as relevant in either explaining a notable amount of variance or accomplishing independent identical normal distributed residuals. Hence, two-level models were sufficient to represent the data structure while preserving parsimony. For all four considered outcome measures, a random intercept and random slope model was deemed appropriate. Because there were only two measurements per outcome, linear change from baseline to follow up was fitted saturated, which is comparable to an analysis of covariance, if no random effects were present.

Data were analyzed with $\mathrm{R}$ environment for statistical computing (Institute for Statistical Computing, Vienna, Austria).

\section{Results}

In total, we sent out 896 invitation letters to GPs. Twentytwo GPs (2\%) agreed to participate after the invitation letters and personal contact. A further eleven GPs agreed to take part in the study after telephone contact, leading to a total number of 33 GPs. No systematic data was collected on the reason for declining, but in the telephone contact, the most common reasons for not taking part were lack of time, interest, and incentives, which is in line with the reasons provided by an earlier study. ${ }^{12}$ The 33 GPs recruited 378 participants meeting the inclusion criteria.
No systematic data was collected about whether invited patients declined to take part in the study, but reasons reported by GPs to the study coordinators were: regarding group exercise as a burden and not enjoyable, low functional status, caring for spouse or other family members or not viewing themselves as at risk of falls. These arguments are in line with other, systematically collected information. ${ }^{9}$ Figure 1 shows the flow of the study.

The characteristics of the patients are presented in Table 2 . In total, 16 trained exercise instructors provided the exercise intervention in 20 groups.

In three of four short-term targeted outcome measures (TUG, mod Rom, FES-I) statistical analysis showed significant differences in the mean change, between the IG and CG. Table 3 shows mean differences in the change obtained from the fitted models beta-coefficients of the interaction terms, with standard errors, Bonferroni-corrected 95\% confidence intervals (CIs), and $P$-values for the four outcome measures. Cohen's $d$ effect sizes were calculated for reasons of comparability. Patients in the IG showed improvement in the TUG that was 1.5 seconds greater than showed by patients in the CG, which is equivalent to a small to moderate effect, as defined by Cohen. ${ }^{23}$ Also in the mod Rom tests, a significant relative improvement, of 0.8 seconds, was accomplished compared with CG. For the CST, no significant differences were found between the IG and CG (1.2 seconds difference).

The overall score of the psychological risk factor - fear of falling - was significantly reduced by 3.7 points in the FES-I questionnaire, in the IG relative to CG.

Mean trajectories in all four considered outcomes of both groups can be viewed in detail in Figure 2 (dotted lines). Box plots represent the distributions of the each of the measures at pretest (T0) and retest (T1).

\section{Adherence to intervention}

In total, $76.6 \%(\mathrm{~N}=170)$ of the IG participated in more than $75 \%$ of the supervised group sessions. Only $2.7 \%(\mathrm{~N}=6)$ missed the supervised training sessions due to sickness. Of all participants, $55.6 \%$ reported that they trained according to the protocol while unsupervised. Of these, $6.6 \%(\mathrm{~N}=15)$ trained unsupervised between one and five times during the intervention phase, $8.6 \%(\mathrm{~N}=19)$ trained six to eight times, $24.3 \%(\mathrm{~N}=55)$ trained nine or ten times, $18 \%(\mathrm{~N}=40)$ trained eleven times, and $12.6 \%$ trained 12 times. No adverse events were reported for the intervention sessions. In three training groups, transportation had to be provided for the participants to take part at each session. 
Start of study

Recruiting GP via post, telephone,

networks and quality circles, and medical

journals
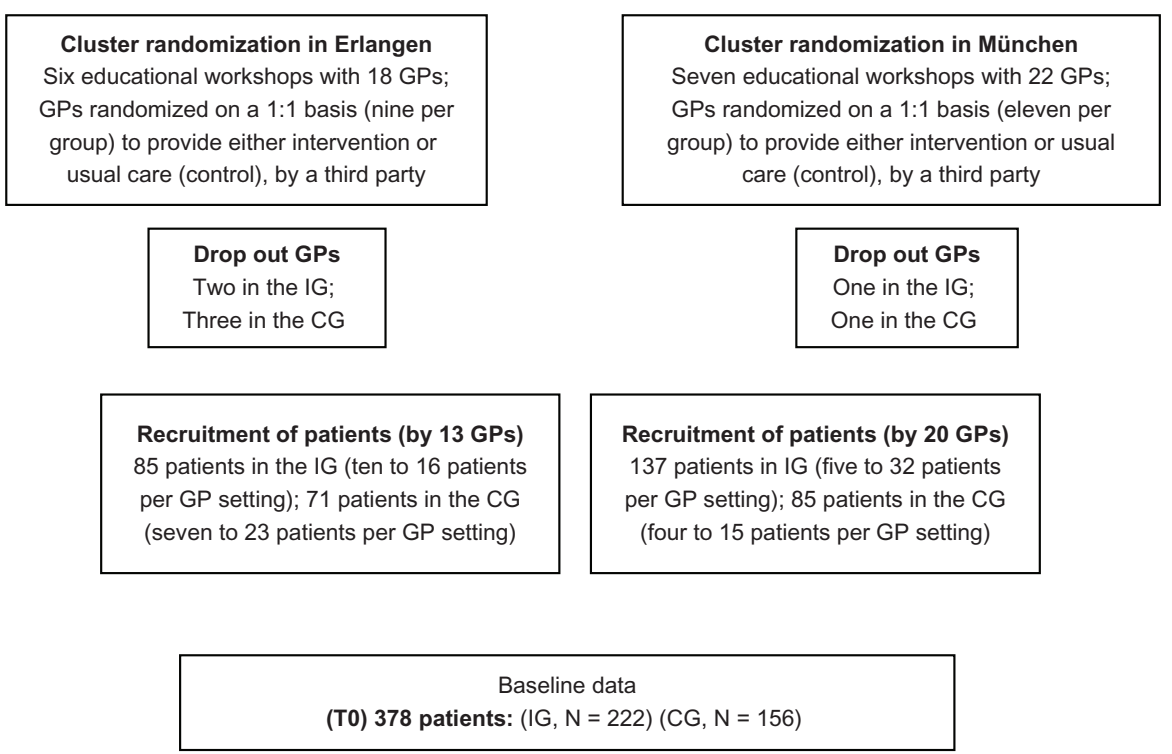

Recruitment of patients (by 20 GPs)

137 patients in IG (five to 32 patients

per GP setting); 85 patients in the CG

(four to 15 patients per GP setting)

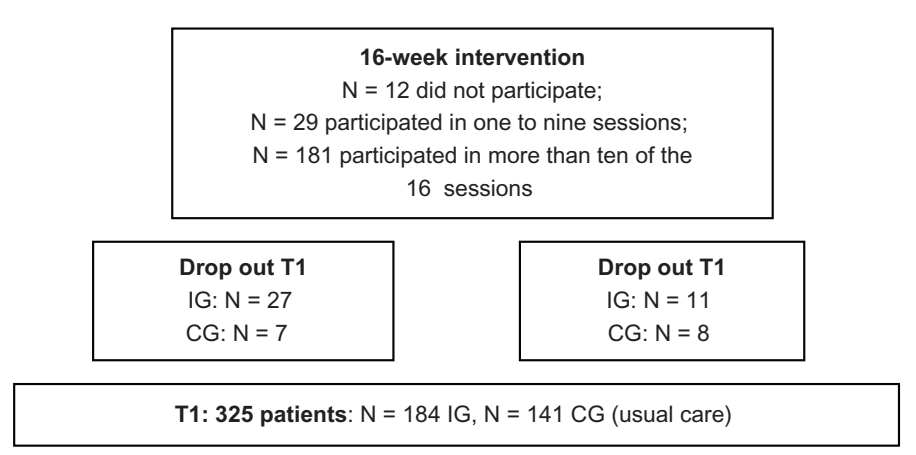

Figure I Flowchart of the study.

Abbreviations: CG, control group; IG, intervention group; GP, general practitioner; T0, baseline; TI, after the intervention.

Table 2 Demographic variables of participants at baseline

\begin{tabular}{lll}
\hline & GP setting & \\
\cline { 2 - 3 } & Intervention & Control \\
\hline Participants (N) & 222 & 156 \\
Age, years (Mean [SD]) & $77.9(5.9)$ & $78.3(5.9)$ \\
Female (\%) & $77.5 \%$ & $72.4 \%$ \\
Living status (\%) & & \\
$\quad$ Alone & $44.1 \%$ & $38.5 \%$ \\
$\quad$ With partner & $38.7 \%$ & $41.0 \%$ \\
Fear of falling at inclusion (\%) & & \\
$\quad$ Not at all/sometimes & $71.2 \%$ & $75.6 \%$ \\
$\quad$ Often/always & $28.0 \%$ & $24.3 \%$ \\
Falls in the last I2 months (\%) & $53.2 \%$ & $51.3 \%$ \\
More than four medications (\%) & $55.4 \%$ & $55.8 \%$ \\
Comorbidities (\%) & & \\
$\quad$ Osteoporosis & $33.3 \%$ & $28.8 \%$ \\
Visual impairments & $41.4 \%$ & $38.5 \%$ \\
$\quad$ Dizziness & $50.2 \%$ & $59.2 \%$ \\
$\quad$ Walking aids & $32.9 \%$ & $43.9 \%$ \\
\hline Abbreviation
\end{tabular}

Abbreviations: GP, general practitioner; SD, standard deviation.

\section{Qualitative data on the intervention by the instructors}

After the end of the intervention, each exercise instructor gave feedback about handling the structured intervention with regard to time management in each exercise session (60 minutes), about the structured protocol of the intervention sessions, and his/her experience with the intervention. The structured protocol of the sessions was rated by $80.2 \%$ of instructors as understandable and easy to handle. In total, $87.5 \%$ rated the time for each session as "just right." The educational aspects included in each session were rated by ten $(62.5 \%)$ of the instructors as "just right." The intensity of the strength, and balance and gait exercise was rated by $13(81.3 \%)$ and $12(75 \%)$, respectively as "just right." The physical capacities of participants improved according to $87.6 \%$ of the exercise instructors. 
Table 3 Results of the random intercept and random slope model for the short-term effects of the intervention on the secondary outcomes

\begin{tabular}{lllllll}
\hline Variable & IG - CG & SE & $\mathbf{9 5 \% ~ C l ~}$ & Cohen's d & P (LRT) & Variable \\
\hline TUG (s) & -1.46 & 0.51 & -2.74 & -0.19 & -0.2 & $0.017^{*}$ \\
CST (s) & -1.19 & 0.98 & -3.64 & 1.27 & -0.08 & 0.896 \\
Romberg (s) & 0.76 & 0.2 & 0.25 & 1.26 & 0.26 & $<0.00 I^{* * *}$ \\
FES-I (scores) & -3.7 & 0.98 & -6.15 & -1.25 & -0.26 & $0.001 * *$ \\
\hline
\end{tabular}

Notes: $* P<0.5$, **P $<0.01$, *** $P<0.001$.

Abbreviations: TUG, Timed-Up-and-Go test; CST, Chair Stand Test; FES-I, Falls Efficacy Scale - International; IG, intervention group; CG, control group; SE, standard error; $\mathrm{Cl}$, confidence interval; LRT, likelihood-ratio-test.

Out of 15 instructors, six gave positive information on continuing the exercise program for the participants, as well as for new participants.

\section{Discussion}

In this paper, we investigated the effects of a 16-week fallprevention intervention on strength, balance, fear of falling, and function, with a cluster, randomized approach, in the GP office setting. With a new approach for fall prevention, we targeted functionally declined, but still independent, community-dwelling older persons by utilizing the GP setting.

Our positive results are in line with other effective exercise interventions addressing risk for falls in functionally declined older persons. ${ }^{1,7,24-26}$ By increasing balance and function and decreasing fear of falling, the most important risk factors for falls have been positively influenced in our study population, who are at high risk of falling. ${ }^{27,28}$

Our intervention showed small to moderate short-term effects on balance (mod Rom), mobility (TUG), and fear of falling (FES-I), but not on strength (CST). Other studies in this population have demonstrated similar results for mobility and balance. ${ }^{7,29-31}$ One explanation for the lack of positive results in strength might be that the exercise was performed - although in a progressive manner - only with body weights and not additional weights. Another explanation for our data could be the enormous variation in physical performance in the IG and CG and/or the delay of effects of our strength training.

Our study supports the design of complex exercise programs, demonstrating effects on physiological and
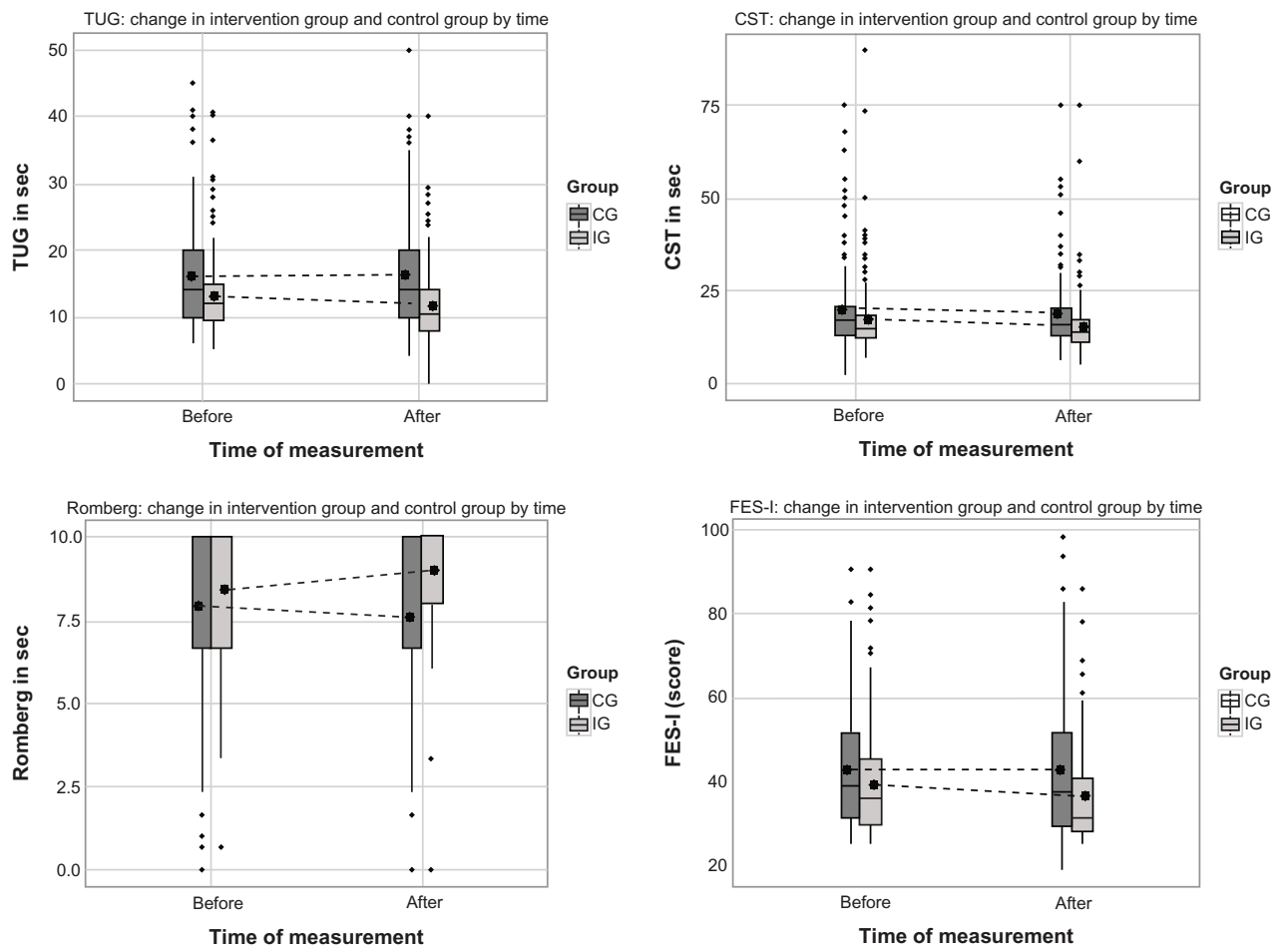

Figure 2 Change in physical and psychological outcomes before and after the intervention.

Abbreviations: CG, control group; CST, Chair Stand Test; FES-I, Falls Efficacy Scale - International; IG, intervention group; TUG, Timed-UP-and-Go test. 
psychological fall risks and thus addressing physical and behavioral dimensions for fall risks. ${ }^{21}$ Although complex interventions, which included educational aspects in addition to the exercise components, make it hard to pinpoint the single effects we demonstrated, addressing individual functional levels with exercise variations seemed valuable to foster adherence and motivation in our study population.

The high adherence rate in our IG was also supported by the provision of transportation possibilities that enabled functionally declined older persons to take part in an exercise intervention program (supporting the findings by McMahon et al). ${ }^{32}$ Our study also demonstrated the feasibility of a combination of supervised and unsupervised sessions in functionally limited, community-dwelling, older persons recruited by their GPs, although we have to admit that information on the unsupervised session was self-reported by the patients, thus to be interpreted with caution. The longitudinal, objective follow-up assessment will give us valuable information on adherence to unsupervised home-based exercise by the participants. The high acceptance and adherence to the structured protocol by the exercise instructors also demonstrates the need for an educational workshop for future instructors, at the start of an intervention. The competence of exercise instructors is essential for the success of the whole study, especially in a multicenter trial, demonstrating the importance of being trained in the underlying concepts and theoretical approaches.

Our strategy to implement a complex fall-prevention exercise program in the GP setting demonstrates the challenges in doing so. In addition to understanding what works, it is necessary to also investigate how implementation in the real world can be achieved. This remains a challenge. ${ }^{33-35}$ Increased awareness of possible strategies to reduce falls and assessing the risk of falling by GPs seems mandatory for positive effects in fall reduction on a larger scale: $:^{8,36}$ GPs are important in fall prevention management due to the acceptance of their advice by older persons ${ }^{9}$ and their role in identifying older at-risk persons. In contrast to other studies, ${ }^{12}$ our study required major efforts to recruit GPs, with an initial response rate of $2 \%$. Although data was not obtained in a structured interview, most GPs declined to participate due to a lack of financial reimbursement and time, or regarded fall prevention as being of little importance. These aspects demonstrate, on the one hand, the need for adequate financial reimbursement if fall prevention strategies are to be implemented successfully in the GP setting and, on the other hand, the ongoing education of GPs with respect to the importance of fall prevention. Nevertheless, our study demonstrated the possibility of implementing fall prevention in the GP setting for older patients. It further supported the need for strategies to raise the awareness in GPs regarding the fall risk in their older patients, eg, educational workshops, familiarization with fall risk assessment, and the importance of a fall definition. ${ }^{4,34,37}$

Nevertheless, some limitations have to be acknowledged. The recruitment of the GPs depended mostly on personal contacts, thus reaching only the already interested GPs. In addition, structured information about the reasons for not participating was not obtained from the invited GPs or patients. Unfortunately, these data were only obtained in telephone contacts in the recruitment process and not followed further. Also, we obtained no information about whether the educational session changed the usual care routine of the control GPs. To our knowledge, the educational session did raise the awareness of regular fall risk screening in all participating GPs, but we were not able to evaluate, with our study, the possible extent of change in daily GP practice.

Another limitation is that the randomization process of the GPs took place before they started recruiting patients, thus causing a problem with the congruency of every variable, despite clear inclusion criteria. In addition, not all randomized GPs were able to recruit participants or lost interest and therefore dropped out the study. In addition, our study had over $70 \%$ female participants, making it hard to generalize our findings to both genders. The last limitation, which has to be mentioned, is the fact that the study was not blinded for the participants, creating a potential bias. However, as has been noted in this paper, it is not possible to solve this problem when comparing an exercise intervention to usual care.

GPs are important persons of trust for older persons, and the advice of GPs is widely recognized. ${ }^{12}$ The strength of our study lies in the strategy of accessing high-risk older persons for fall prevention through their GPs, with a complex approach targeting the biopsychosocial resources of the participants.

\section{Conclusion}

The new strategy to target highly at-risk and functionally declined community-dwelling, older persons for fall prevention via a GP setting seemed promising. Our complex exercise intervention for fall prevention has effectively improved balance, physical function, and led to a reduction in fear of falling in this population. Further research must investigate the process of further maintenance and adherence, as well as the longitudinal effects of the exercise program. 


\section{Author contributions}

Ellen Freiberger participated in study conceptualization and design, acquisition of subjects and/or data, intervention development, analysis and interpretation of data, and wrote major parts of the manuscript. Wolfgang A Blank participated in study conceptualization and design, acquisition of subjects and/or data, and manuscript preparation. Johannes Salb participated in acquisition of subjects and/or data, and manuscript preparation. Barbara Geilhof participated in acquisition of subjects and/or data, and manuscript preparation. Christian Hentschke participated in analysis and interpretation of data, and he drafted the tables and figures, and prepared the statistical part of the manuscript. Peter Landendörfer participated in study conceptualization and design, and acquisition of subjects and/or data. Martin Halle participated in study conceptualization and design, and manuscript preparation. Monika Siegrist participated in study conceptualization and design, acquisition of subjects and/or data, intervention development, analysis and interpretation of data, and manuscript preparation.

\section{Acknowledgment}

This work was funded by a grant from the Bavarian State Ministry of the Environment and Public Health (Gesund. Leben. Bayern.) (Grant number: LP 00110, Pr Nr 09-10).

We want to thank Liz Woodward for reviewing our paper as a native speaker, and we want to thank all our participants as well as the general practitioners and their staff, for taking part in our study and giving us their time.

\section{Disclosure}

The authors report no conflicts of interest in this work.

\section{References}

1. Gillespie LD, Robertson MC, Gillespie WJ, et al. Interventions for preventing falls in older people living in the community. Cochrane Database Syst Rev. 2012;9:CD007146.

2. Tinetti M, Williams CS. Falls, injuries due to falls, and the risk of admission to a nursing home. $N$ Engl J Med. 1997;337(18):1279-1284.

3. Kannus P, Sievänen H, Palvanen M, Järvinen T, Parkkari J. Prevention of falls and consequent injuries in elderly people. Lancet. 2005;366(9500):1885-1893.

4. Rubenstein LZ, Solomon DH, Roth CP, et al. Detection and management of falls and instability in vulnerable elders by community physicians. J Am Geriatr Soc. 2004;52(9):1527-1531.

5. Sattin RW. Falls among older persons: a public health perspective. Annu Rev Public Health. 1992;13:489-508.

6. Day L, Finch CF, Hill KD, et al. A protocol for evidence-based targeting and evaluation of statewide strategies for preventing falls among community-dwelling older people in Victoria, Australia. Inj Prev. 2011;17(2):e3.

7. Sherrington C, Tiedemann A, Fairhall N, Close JC, Lord SR. Exercise to prevent falls in older adults: an updated meta-analysis and best practice recommendations. N S W Public Health Bull. 2011;22(3-4):78-83.
8. Ganz DA, Alkema GE, Wu S. It takes a village to prevent falls: reconceptualizing fall prevention and management for older adults. Inj Prev. 2008;14(4):266-271.

9. Yardley L, Donovan-Hall M, Francis K, Todd C. Attitudes and beliefs that predict older people's intention to undertake strength and balance training. J Gerontol B Psychol Sci Soc Sci. 2007;62(2):P119-P125.

10. Day L, Fildes B, Gordon I, Fitzharris M, Flamer H, Lord S. Randomised factorial trial of falls prevention among older people living in their own homes. BMJ. 2002;325(7356):128.

11. Yardley L, Kirby S, Ben-Shlomo Y, Gilbert R, Whitehead S, Todd C. How likely are older people to take up different falls prevention activities? Prev Med. 2008;47(5):554-558.

12. Gardner MM, Phty M, Robertson MC, McGee R, Campbell AJ. Application of a falls prevention program for older people to primary health care practice. Prev Med. 2002;34(5):546-553.

13. Gates S, Fisher JD, Cooke MW, Carter YH, Lamb SE. Multifactorial assessment and targeted intervention for preventing falls and injuries among older people in community and emergency care settings: systematic review and meta-analysis. $B M J$. 2008;336(7636):130-133.

14. Deutsche Gesellschaft für Allgemeinmedizin (DEGAM). Ältere Sturzpatienten DEGAM Leitlinien Nr. 4. 2004. Accessed Dec. 2004. German.

15. Muller CA, Klaassen-Mielke R, Penner E, Junius-Walker U, Hummers-Pradier E, Theile G. Disclosure of new health problems and intervention planning using a geriatric assessment in a primary care setting. Croat Med J. 2010;51(6):493-500.

16. Blank WA, Freiberger E, Siegrist M, et al. An interdisciplinary intervention to prevent falls in community-dwelling elderly persons: protocol of a cluster-randomized trial [PreFalls]. BMC Geriatr. $2011 ; 11: 7$

17. Shumway-Cook A, Brauer S, Woollacott M. Predicting the probability for falls in community-dwelling older adults using the Timed Up \& Go Test. Phys Ther. 2000;80:896-903.

18. Guralnik J, Ferrucci L, Pieper CF, et al. Wallace RB. Lower extremity function and subsequent disability: consistency across studies, predictive models, and value of gait speed alone compared with the short physical performance battery. J Gerontol A Biol Sci Med Sci, 2000;55(4):221-M231.

19. Craig P, Dieppe P, Macintyre S, et al; Medical Research Council Guidance. Developing and evaluating complex interventions: the new Medical Research Council guidance. BMJ. 2008;337:a1655.

20. Freiberger E, Menz HB, Abu-Omar K, Rutten A. Preventing falls in physically active community-dwelling older people: a comparison of two intervention techniques. Gerontology. 2007;53(5): 298-305.

21. Freiberger E, Häberle L, Spirduso WW, Zijlstra GA. Long-term effects of three multicomponent exercise interventions on physical performance and fall-related psychological outcomes in communitydwelling older adults: a randomized controlled trial. J Am Geriatr Soc. 2012;60(3):437-446.

22. Borg G. Borg's Perceived Exertion and Pain Scales. Champaign, IL, USA: Human Kinetics; 1998.

23. Cohen J. Statistical Power Analysis for the Behavioral Sciences. Hillsdale, NJ: Lawrence Erlbaum Associates; 1988.

24. Liu-Ambrose T, Khan KM, Eng JJ, Janssen PA, Lord SR, McKay HA. Resistance and agility training reduce fall risk in women aged 75 to 85 with low bone mass: a 6-month randomized, controlled trial. $\mathrm{J} \mathrm{Am}$ Geriatr Soc. 2004;52(5):657-665.

25. Liu-Ambrose T, Khan KM, Eng JJ, Lord SR, McKay HA. Balance confidence improves with resistance or agility training. Increase is not correlated with objective changes in fall risk and physical abilities. Gerontology. 2004;50(6):373-382.

26. Alfieri FM, Riberto M, Abril-Carreres A, et al. Effectiveness of an exercise program on postural control in frail older adults. Clin Interv Aging. 2012;7:593-598. 
27. Todd C, Skelton D. What are the Main Risk Factors for Falls Among Older People and What are the Most Effecxtive Interventions to Prevent these Falls? Copenhagen: WHO Regional Office for Europe (Health Evidence Network Report); 2004. Available from: http://www.euro. who.int/document/e82552.pdf. Accessed June 26, 2013.

28. Tinetti ME, Speechley M, Ginter SF. Risk factors for falls among elderly persons living in the community. $N$ Engl J Med. 1988;319(26):1701-1707.

29. Bulat T, Hart-Hughes S, Ahmed S, et al. Effect of a group-based exercise program on balance in elderly. Clin Interv Aging. 2007;2(4): 655-660.

30. Faber MJ, Bosscher RJ, Chin A, Paw MJ, van Wieringen PC. Effects of exercise programs on falls and mobility in frail and pre-frail older adults: A multicenter randomized controlled trial. Arch Phys Med Rehabil. 2006;87(7):885-896.

31. DeVito CA, Morgan RO, Duque M, Abdel-Moty E, Virnig BA. Physical performance effects of low-intensity exercise among clinically defined high-risk elders. Gerontology. 2003;49(3):146-154.
32. McMahon S, Talley KM, Wyman JF. Older people's perspectives on fall risk and fall prevention programs: a literature review. Int J Older People Nurs. 2011;6(4):289-298.

33. Ganz DA, Yano EM, Saliba D, Shekelle PG. Design of a continuous quality improvement program to prevent falls among community-dwelling older adults in an integrated healthcare system. BMC Health Serv Res. 2009;9:206.

34. Tinetti ME, Baker DI, King M, et al. Effect of dissemination of evidence in reducing injuries from falls. $N$ Engl J Med. 2008;359(3):252-261.

35. Hendriks MR, Bleijlevens MH, van Haastregt JC, et al. Lack of effectiveness of a multidisciplinary fall-prevention program in elderly people at risk: a randomized, controlled trial. J Am Geriatr Soc. 2008;56(8): 1390-1397.

36. Tinetti ME, Gordon C, Sogolow E, Lapin P, Bradley EH. Fall-risk evaluation and management: challenges in adopting geriatric care practices. Gerontologist. 2006;46(6):717-725.

37. Jette AM. "Invention is hard, but dissemination is even harder". Phys Ther. 2005;85(5):390-391.
Clinical Interventions in Aging

\section{Publish your work in this journal}

Clinical Interventions in Aging is an international, peer-reviewed journal focusing on evidence-based reports on the value or lack thereof of treatments intended to prevent or delay the onset of maladaptive correlates of aging in human beings. This journal is indexed on PubMed Central, MedLine, the American Chemical Society's 'Chemical Abstracts

\section{Dovepress}

Service' (CAS), Scopus and the Elsevier Bibliographic databases. The manuscript management system is completely online and includes a very quick and fair peer-review system, which is all easy to use. Visit http://www.dovepress.com/testimonials.php to read real quotes from published authors. 